\title{
On the characterization of battery electrolytes with polarization cells
}

\author{
Jing Liu, Charles W. Monroe* \\ Department of Chemical Engineering, University of Michigan, Ann Arbor, MI 48109, USA \\ Joint Center for Energy Storage Research, University of Michigan, Ann Arbor, MI 48109, USA
}

\section{Abstract}

The theoretical foundation of potentiometric diffusivity and transference-number measurements is revisited, through an analysis of the diffusion-potential relaxation a concentrated binary electrolyte exhibits after being subjected to a current pulse in a planar electrolytic cell. Earlier theory is extended to include solute-volume effects, as well as being modified to incorporate a particle-fraction basis for composition and to account for the nonlinear relationship between composition differences and cell voltage. The new theory provides significant corrections when concentration polarization is very large or when electrolytes are moderately concentrated, rationalizing the unexpected voltage responses seen during some previous transport measurements. Guidelines are developed to aid the design of galvanostatic-polarization experiments involving non-aqueous electrolytes similar to those used in lithium batteries. Complete property sets are provided for $0.85 \mathrm{M} \mathrm{LiPF}_{6}$ in propylene carbonate and for $2.24 \mathrm{M} \mathrm{LiPF}_{6}$ in a mixed-carbonate solvent.

Keywords: restricted diffusion, galvanostatic polarization, concentrated-solution theory transport coefficients, salt diffusivity, transference number

\section{Introduction}

The proper description of mass transfer requires that a transport property be associated with every pair of chemically distinct species a phase contains [1]. Since a binary electrolytic solution comprises cations, anions, and a solvent, three properties are needed. The ionic conductivity $\kappa$, the cation transference number relative to the solvent velocity $t_{+}^{0}$, and the thermodynamic diffusivity $\mathscr{D}$ provide a sufficient set of fundamental transport parameters [2].

Several thermodynamic properties are also required to complete the macroscopic description of an isothermal, isobaric, multicomponent phase. A set of thermodynamic factors must be quantified to establish how changes in a solution's composition relate to changes in its free energy; an additional equation of state may also be adopted to account for how a solution's density depends on its composition. For an electroneutral binary electrolytic solution, there is a single thermodynamic factor, $\chi$, and the state equation involves the partial molar volumes of the solvent and dissolved electrolyte, $\bar{V}_{0}$ and $\bar{V}_{\mathrm{e}}$, respectively. Thermodynamic factors are particularly important because solution nonideality can be significant [3]. Also, the time scale over which concentration differences relax is determined by the Fickian diffusivity $D=\mathscr{D} \chi$, rather than $\mathscr{D}$ alone [4].

\footnotetext{
*Corresponding author.

Email addresses: liujin@umich.edu (Jing Liu), cwmonroe@umich.edu (Charles W. Monroe)

Ionic conductivity is directly measurable, and is typically quantified using DC conductometry $[5,6]$ or $\mathrm{AC}$ impedance spectroscopy $[7,8]$. Partial molar volumes are not directly measurable, but their composition dependences can be quantified independently of the other properties via densitometry $[9,10]$. The other three solution properties are not directly measurable, and cannot be measured in isolation; values of $\mathscr{D}, t_{+}^{0}$, and $\chi$ are usually obtained by simultaneously analyzing results from several experiments.

Ma et al. proposed a suite of three potentiometric experiments to measure $\mathscr{D}, t_{+}^{0}$, and $\chi[11]$, which all involve cells with two electrochemically reversible electrodes that produce or consume species from the solution phase. One measurement is performed with a 'concentration cell', in which similar binary electrolytes with different salt concentrations are put in chemical contact through a porous membrane that prevents significant mixing during the experimental period; the open-circuit potential (OCP) across a quasiequilibrated concentration cell relates to $\chi$ and $t_{+}^{0}$ $[2,3,12]$. The other two measurements are performed using a 'polarization cell': a planar electrolytic cell with parallel electrodes that allow a current to be driven through the electrolyte between them. In a 'restricted diffusion' measurement, an applied current is used to induce a nonuniform concentration distribution in the interelectrode gap; the relaxation at long times is exponential, with a time constant dependent on $\mathscr{D}$ and $\chi[4,13]$ that can be tracked by recording the transient OCP $[4,14]$. In a 'galvanostatic polarization' measurement, a short current pulse is applied to produce diffusion boundary layers 
near the electrode surfaces; the OCP relaxation at times before the boundary layers come into contact depends on $\mathscr{D}, t_{+}^{0}$, and $\chi[11,15]$. The two polarization-cell measurements can in principle be implemented during a single experiment, since the current pulse that initiates the galvanostatic polarization can be used to establish the nonuniform initial concentration for restricted diffusion. In practice, separate experiments may be needed to achieve good signal-to-noise ratios for voltages in the short-time and long-time relaxation regimes.

Theoretical expressions derived by Ma et al. [11] relate the voltage transients during restricted-diffusion and galvanostatic-polarization experiments to $\mathscr{D}, t_{+}^{0}$, and $\chi$. A number of restrictions apply to their analysis. The theory requires that concentration differences are relatively small, despite the fact that large differences may be needed to get an adequate chronopotentiometry signal. Electrolyte convection associated with current pulses is also neglected - but this phenomenon can sometimes be significant for moderately concentrated non-aqueous electrolytes [16].

Although $\mathrm{Ma}$ et al. reported sensible property values [11], some implementations of their protocol by others have led to surprising results for lithium-battery electrolytes. These include unexpectedly high diffusivities [12] and low - or even negative - transference numbers [17]. In light of these observations, the present discussion has three main aims. First, the theory underpinning potentiometric measurement of $\mathscr{D}, t_{+}^{0}$, and $\chi$ is extended, to relax the assumptions of low concentration polarization and negligible solute-volume effects. Second, the constraints on pulse currents are analyzed to guide experimentalists in the design of galvanostatic-polarization measurements. Third, the extended theory is used to rationalize some counterintuitive observations made by prior researchers. In the course of the discussion, complete property sets are developed for two typical lithium-battery electrolytes.

\section{Thermodynamic fundamentals}

\subsection{Electrolyte composition bases}

Solute molalities $m_{k}$ are the most typical composition basis used to express the composition dependences of solution properties, primarily because they are very precisely quantifiable. To prepare a solution of a given molality does not require a volume measurement, and, since it is referred to solvent mass, molality is independent of temperature $T$ and pressure $p$. Although a solvent's molality is conveniently constant (equaling the inverse of its molar mass $M_{0}$ ), the molalities of solutes become very large when little solvent is present, and diverge in the limit where there is no solvent. Thus molality is a difficult composition basis to use for very concentrated solutions. The need to specially identify a particular species as a 'solvent' also forces some degree of arbitrariness when describing the mixedsolvent solutions commonly used for lithium batteries.

Molar species concentrations $c_{k}$ are finite throughout the entire domain of miscibility and treat all species on

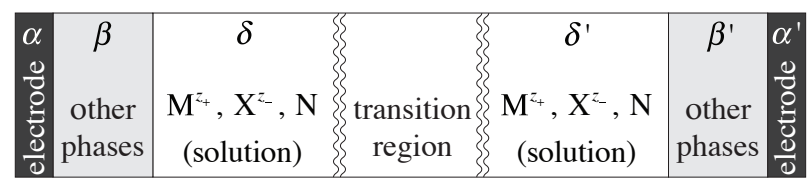

Figure 1: Schematic drawing of a general electrolytic cell containing a binary electrolytic solution.

equal footing, avoiding the problems associated with molality in very concentrated or multi-solvent systems. The molar basis places strict limits on experimental precision, however, because solution preparation involves a volume measurement. Since solution densities depend on $T$ and $p$, as well as local composition, the use of a molar basis can easily lead to systematic measurement errors. The $T$ and $p$ dependences of molarity also make it vague as a basis for properties in coupled transport scenarios where heat or momentum transfer accompanies mass transfer.

Species particle fractions $y_{k}$ have the advantage of molality, because they are independent of $T$ and $p$, and also of molarity, because they apply equitably to every species in a phase and are always finite. They are convenient from a theoretical standpoint because they are dimensionless and bounded between 0 and 1 . As a basis for activity they are further useful, because chemical-potential constitutive laws based on particle fractions satisfy the Gibbs-Duhem equation naturally in the ideal-solution limit, where every species activity coefficient approaches 1 .

When bulk electrolytic solutions are observed on the length scales relevant to macroscopic transport phenomena, their ion contents satisfy a local electroneutrality relationship. Thus one usually does not need to distinguish the cation particle fraction $y_{+}$and the anion particle fraction $y_{-}$in a binary electrolytic solution. Letting $\nu_{+}$and $\nu_{-}$ represent the ion stoichiometries in a formula unit of salt, one can instead use a single composition variable

$$
y=\frac{y_{+}}{\nu_{+}}=\frac{y_{-}}{\nu_{-}} .
$$

This quantity represents the number of salt formula units, scaled by the total number of ion and solvent particles (i.e., the sum of the amounts of all three species) in the solution. The particle-fraction basis relates to salt molality $m$ and salt molarity $c$ through

$$
y(m)=\frac{m M_{0}}{1+\nu m M_{0}} \text { and } y(c)=\frac{c \bar{V}_{0}}{1+c\left(\nu \bar{V}_{0}-\bar{V}_{\mathrm{e}}\right)},
$$

in which $\nu=\nu_{+}+\nu_{-}$stands for the total number of ions in a formula unit.

\subsection{The open-circuit potential}

Consider a general electrolytic cell containing a binary electrolytic solution, diagrammed using the standard schematic representation $[2,18]$ in Figure 1. Half-reactions 
at each of the electrodes can be expressed in the form

species in electrode \& other phases

$$
+s_{+} \mathrm{M}^{z_{+}}+s_{-} \mathrm{X}^{z_{-}}+s_{0} \mathrm{~N} \rightleftharpoons n_{\mathrm{e}^{-}} \mathrm{e}^{-}
$$

in which $\mathrm{M}, \mathrm{X}$, and $\mathrm{N}$ are the chemical symbols of cations, anions, and solvent, respectively, and $z_{+}$and $z_{-}$are the ionic equivalent charges. The cell is taken to be 'symmetric', in the sense that the stoichiometric coefficients $s_{k}$ for all liquid-phase species ${ }^{1}$ are the same in both electrode reactions (the electron exchanges at $\alpha$ and $\alpha^{\prime}$ ); the number of electrons transferred, $n_{\mathrm{e}^{-}}$, should also equate. One also needs to stipulate that the species in the electrodes and other phases are uncharged, in which case conservation of charge requires that $\sum_{k} s_{k} z_{k}=n_{\mathrm{e}^{-}} z_{\mathrm{e}^{-}}$, where $z_{\mathrm{e}^{-}}=-1$ is the equivalent charge of electrons. Despite these restrictions, the fabrication of a symmetric electrolytic cell does not require that both half-reactions are the same: the components in other phases (if present) and their involvement in the electrode reactions can generally be dissimilar. ${ }^{2}$

When no current is allowed to pass through the cell in Figure 1, the diffusion potential arising from the composition change across the transition region, $U_{\mathrm{c}}$, is given by the path integral

$$
F U_{\mathrm{c}}=\frac{s_{+}}{n_{\mathrm{e}^{-}}} \int_{\delta}^{\delta^{\prime}} d \mu_{+}+\frac{s_{-}}{n_{\mathrm{e}^{-}}} \int_{\delta}^{\delta^{\prime}} d \mu_{-}+\frac{s_{0}}{n_{\mathrm{e}^{-}}} \int_{\delta}^{\delta^{\prime}} d \mu_{0} .
$$

Here $\mu_{k}$ represents the electrochemical potential of species $k$ and $F$ is Faraday's constant. Equation 4 for the OCP difference holds true so long as an unbroken path of chemical contact can be traversed from $\delta$ to $\delta$ '. The 'transition region' in Figure 1 is intended to represent any means by which this continuous contact is supported: it can be a fabricated liquid junction (e.g., a porous glass frit or membrane), or simply a nonuniform composition distribution in the intermediating solution between the electrodes. It should be emphasized that $U_{\mathrm{c}}$ differs from the OCP, $U$, which formally quantifies the energy difference between electrons in $\alpha$ and $\alpha^{\prime}: U=U_{\mathrm{c}}+U_{\mathrm{rxn}}$. The additional factor $U_{\text {rxn }}$ is constant if the compositions in $\alpha, \alpha^{\prime}, \beta$ and $\beta^{\prime}$ do not change over time at open circuit. In this situation, $U$ and $U_{\mathrm{c}}$ equate if the cell half-reactions are identical, but $U_{\text {rxn }}$ is nonzero otherwise [2].

Every integration variable in equation 4 depends only on $y$ at constant $T$ and $p$. Following Newman and ThomasAlyea [2], the salt dissociation equilibrium $\mu_{\mathrm{e}}=\nu_{+} \mu_{+}+\nu_{-} \mu_{-}$ can be used to eliminate $\mu_{-}$; the isothermal, isobaric, electroneutral Gibbs-Duhem equation, $y d \mu_{\mathrm{e}}+y_{0} d \mu_{0}=0$, then allows elimination of $\mu_{\mathrm{e}}$. Changes in the solvent chemical

${ }^{1}$ The coefficient $s_{k}$ is positive for a product and negative for a reactant when half-reaction 3 is written as a reduction.

${ }^{2}$ For example, an alkaline battery is a symmetric cell: although one electrode is zinc and one is manganese oxide, both half-reactions involve the same number of electrons, and both electrodes react with solvent (water) and anions (hydroxide) in similar proportions. potential relate to composition through a constitutive law that defines $\chi[16]$,

$$
d \mu_{0}=-\frac{\nu R T \chi}{y_{0}} d y
$$

so terms involving $\mu_{0}$ can be replaced with terms involving $y$. Incorporation of the MacInnes equation in the form given recently by the authors [16], under conditions of zero current, allows $d \mu_{+}$to be eliminated in favor of $d y$ as well. Thus $U_{\mathrm{c}}$ is a functional of $y$ alone, and because it differs from $U$ by a constant, it follows that

$$
\begin{array}{r}
\int_{\delta}^{\delta^{\prime}}\left\{\frac{d U}{d \ln y}-\frac{\nu R T \chi}{F}\left[\frac{s_{+}}{n_{\mathrm{e}^{-} \nu_{+}}}-\frac{z_{\mathrm{e}^{-}} t_{+}^{0}}{z_{+} \nu_{+}}\right.\right. \\
\left.\left.-\frac{s_{0} y}{n_{\mathrm{e}^{-}}(1-\nu y)}\right]\right\} d \ln y=0 .
\end{array}
$$

This relationship holds along any portion of the path between $\delta$ and $\delta^{\prime}$, so the integrand vanishes uniformly, and

$$
\left(\frac{d U}{d \ln y}\right)_{T, p}=\frac{\nu R T \chi}{F}\left[\frac{s_{+}}{n_{\mathrm{e}^{-} \nu_{+}}}-\frac{z_{\mathrm{e}^{-} t_{+}^{0}}}{z_{+} \nu_{+}}-\frac{s_{0} y}{n_{\mathrm{e}^{-}}(1-\nu y)}\right] .
$$

Equation 7 differs from the expression used by Ma et al. [11] because it involves a particle-fraction basis, rather than being based on molar concentration.

Many electrolytes can be treated as 'simple binary electrolytic solutions' [16], for which $\mathscr{D}, t_{+}^{0}, \chi$, and the $\bar{V}_{k}$ (but not $\kappa)$ are constant with composition. In this case equation 7 can be integrated directly, yielding

$$
\Delta U=\frac{R T \chi}{F}\left[\frac{\nu}{\nu_{+}}\left(\frac{s_{+}}{n_{\mathrm{e}^{-}}}-\frac{z_{\mathrm{e}^{-}} t_{+}^{0}}{z_{+}}\right) \ln \frac{y^{\delta^{\prime}}}{y^{\delta}}+\frac{s_{0}}{n_{\mathrm{e}^{-}}} \ln \frac{1-\nu y^{\delta^{\prime}}}{1-\nu y^{\delta}}\right] .
$$

This is the sole expression needed to determine changes in the OCP arising from composition variation, $\Delta U$, when a simple binary electrolytic solution is placed in a symmetric electrolytic cell. It provides the theoretical groundwork for the subsequent analysis of potentiometric measurements.

\subsection{Linearization of potential}

Potentiometric restricted-diffusion and galvanostaticpolarization measurements both use $\Delta U$ as a proxy for the composition difference across the cell. If the magnitude of concentration polarization is sufficiently small, the OCP can be linearized about the equilibrium composition:

$$
\left.\Delta U \approx \frac{d U}{d y}\right|_{\langle y\rangle} \Delta y, \quad \text { or }\left.\Delta U \approx \frac{d U}{d \ln y}\right|_{\langle y\rangle} \frac{\Delta y}{\langle y\rangle},
$$

where $\Delta y=y^{\delta^{\prime}}-y^{\delta}$. This proportionality between $\Delta U$ and $\Delta y$ has been adopted instead of equation 8 in the analyses of many electrolyte-characterization experiments $[3,11$, $12,15,17,19-21]$; several groups have employed equation 9 when implementing diffusivity and transference-number measurements in particular $[3,17,19,20]$. One should be aware that the restriction to small $\Delta y$ can easily be violated in practice, because large concentration differences may be needed to achieve a significant voltage signal during a relaxation experiment. 


\section{Characterization experiments}

\subsection{Concentration cell}

During concentration-cell measurements, the cell voltage is recorded while no appreciable mass diffusion is allowed across the transition region in Figure 1. Experimental implementations typically involve using a membrane or frit to separate two compartments containing well-stirred electrolytic solutions, in which reference electrodes are immersed. On one side of the cell, solution composition is fixed at $y^{\delta^{\prime}}=y_{\text {ref }}$; on the other side, a test composition $y^{\delta}=y_{\text {test }}$ is varied in the close vicinity of $y_{\text {ref }}$. The potential difference between the two electrodes is determined by $y_{\text {test }}$ and $y_{\text {ref }}$, giving a function $\Delta U\left(y_{\text {test }}, y_{\text {ref }}\right)$. Plotting this potential difference as a function of the logarithm of the concentration ratio $y_{\text {test }} / y_{\text {ref }}$ yields a curve, whose slope can be extrapolated to find the value of $d U / d \ln y$ at $y_{\text {ref }}$, denoted formally as $\left.(d U / d \ln y)\right|_{y_{\text {ref }}}[11]$. Given the tranference number at $y_{\text {ref }}$, the thermodynamic factor at $y_{\text {ref }}$ can be calculated from $\left.(d U / d \ln y)\right|_{y_{\text {ref }}}$ through equation 7 , or vice versa.

\subsection{Polarization cell}

Restricted diffusion and galvanostatic polarization both involve tracking the relaxation of an initially nonuniform concentration distribution in the transition region of Figure 1 under open-circuit conditions $[4,11,21]$. Both measurements can be implemented by pulsing a symmetric electrolytic cell with a constant current for a period $t_{\text {pulse }}$ to induce the nonuniform concentration. After $t_{\text {pulse }}$, an open-circuit condition can be enforced by a galvanostat, which also records the relaxation of the OCP as the concentration profile relaxes.

For a simple binary electrolyte in a one-dimensional planar cell of length $L$ in the $x$ direction, the transient composition distribution is governed by [16]

$$
\frac{\partial y}{\partial \tau}+2 \beta I \frac{\partial y}{\partial \xi}=\frac{\partial^{2} y}{\partial \xi^{2}}+\frac{2 \alpha}{\langle y\rangle+(\langle y\rangle-y) \alpha}\left(\frac{\partial y}{\partial \xi}\right)^{2},
$$

where $\langle y\rangle$ is the equilibrium composition. The dimensionless time $\tau$, position $\xi$, and current $I$ are defined as

$$
\tau=\frac{\mathscr{D} \chi t}{L}, \quad \xi=\frac{x}{L}, \quad \text { and } \quad I=\frac{i}{i_{\mathrm{L}}^{\infty}}
$$

and the limiting current density at infinite dilution, $i_{\mathrm{L}}^{\infty}$, is

$$
i_{\mathrm{L}}^{\infty}=\frac{2 F z_{+} \nu_{+} \mathscr{D} \chi\langle c\rangle}{\left(\frac{s_{+} z_{+}}{z_{\mathrm{e}^{-}} n_{\mathrm{e}^{-}}}-t_{+}^{0}\right) L} .
$$

(The equilibrium molar concentration of salt, $\langle c\rangle$, relates to $\langle y\rangle$ through equation 2.) For binary electrolytes, $\mathscr{D}$ and $t_{+}^{0}$ are defined in terms of the Stefan-Maxwell coefficients describing ion-solvent interactions as

$$
\mathscr{D}=\frac{\left(z_{+}-z_{-}\right) \mathscr{D}_{0+} \mathscr{D}_{0-}}{z_{+} \mathscr{D}_{0+}-z_{-} \mathscr{D}_{0-}} \quad \text { and } \quad t_{+}^{0}=\frac{z_{+} \mathscr{D}_{0+}}{z_{+} \mathscr{D}_{0+}-z_{-} \mathscr{D}_{0-}} .
$$

In equation 10, solute-volume effects are described by the parameters

$$
\alpha=\langle c\rangle\left(\nu \bar{V}_{0}-\bar{V}_{\mathrm{e}}\right) \text { and } \beta=\frac{z_{+} \nu_{+}\langle c\rangle \Delta \bar{V}_{\mathrm{rxn}}^{\mathrm{sol}}}{z_{+} s_{+}-z_{\mathrm{e}^{-}} n_{\mathrm{e}^{-}} t_{+}^{0}},
$$

in which $\Delta \bar{V}_{\mathrm{rxn}}^{\mathrm{sol}}=\sum_{k} s_{k} \bar{V}_{k}$ represents the solution-phase volume change per mole of limiting reactant as reaction 3 goes to completion. Representative values of $\alpha$ and $\beta$ are provided in our earlier communication [16], which also provides a deeper discussion of their physical significance.

Observe that the computation of $\beta$ can involve the partial molar volumes of individual ions. Newman and Chapman [4] showed for binary electrolytic solutions that it is natural to assume

$$
\bar{V}_{+}=\frac{\left(1-t_{+}^{0}\right) \bar{V}_{\mathrm{e}}}{\nu_{+}} \text {and } \bar{V}_{-}=\frac{t_{+}^{0} \bar{V}_{\mathrm{e}}}{\nu_{-}},
$$

which can be used to calculate $\Delta \bar{V}_{\mathrm{rxn}}^{\mathrm{sol}}$.

Because of the interfacial half-reaction, governing equation 10 is subject to a boundary condition

$$
\begin{aligned}
& \left.\frac{\partial y}{\partial \xi}\right|_{\tau, 0}=-\frac{2\langle y\rangle}{1+\alpha} \times \\
& \left.\quad\left\{\left[1+\left(1-\frac{y}{\langle y\rangle}\right) \alpha-\frac{y}{\langle y\rangle} \beta\right]\left[1+\left(1-\frac{y}{\langle y\rangle}\right) \alpha\right] I\right\}\right|_{\tau, 0},
\end{aligned}
$$

so the composition gradient at one of the electrodes relates to the dimensionless current $I$. To describe the pulse process, current is expressed in terms of a driving function

$$
I(\tau)=\left\{\begin{array}{ll}
I_{\text {pulse }}, & 0 \leq \tau \leq \tau_{\text {pulse }} \\
0, & \tau>\tau_{\text {pulse }}
\end{array} .\right.
$$

In addition, an auxiliary condition,

$$
\int_{0}^{1} \frac{y}{\langle y\rangle+(\langle y\rangle-y) \alpha} d \xi=1
$$

guarantees mass conservation in the solution phase, which is maintained continuously as a consequence of the symmetry in the cell half-reactions.

The solution of equation 10 subject to the conditions expressed by equations 16 through 18 describes how a galvanostatic pulse induces concentration polarization, and how that polarization subsequently relaxes at open circuit. Even when transient diffusional relaxations are occuring, equation 8 determines the instantaneous OCP. The values of $y^{\delta}$ and $y^{\delta^{\prime}}$ are taken to be the transient solution compositions in domains immediately adjacent to the the electrode surfaces (i.e., $y^{\delta^{\prime}}=y(\tau, 0)$ and $y^{\delta}=y(\tau, 1)$ ), which in this case are determined by the pulse current and duration.

Note that the assumption of a simple binary electrolyte is not necessary, although it is justified for several systems [16]. The approximation is applied to the electrolytes here first for simplicity, and second, because it is found to be 
able to match experimental diffusion-potential transients very well. To relax the approximation, one can use the transport equations given in section 2.7 of reference [16], which do not require constant properties, when determining concentration distributions. In this case $t_{+}^{0}$ and $\chi$ must also be taken to depend on local concentration when integrating equation 7 to obtain the diffusion potential.

\subsection{Restricted diffusion}

Restricted-diffusion measurements track OCP relaxations long after a concentration polarization - a time domain when migration is unimportant and detailed information about the initial distribution of composition is lost. In this situation the timescale for relaxation depends on the Fickian diffusivity and the electrode spacing.

To implement a restricted-diffusion measurement, an initially nonuniform concentration distribution is set up and the logarithm of the concentration difference between ends of the cell (or the difference in some other local solution characteristic that is proportional to concentration) is tracked as a function of time. Data gathered at long times are fit with a line, whose slope is proportional to the Fickian diffusivity $D=\mathscr{D} \chi$.

Although OCP is a logical solution characteristic to track, restricted-diffusion measurements can be performed in a number of ways that do not involve potentiometry. For example, the classic Harned technique for measuring $D$ relies on local conductometry to establish concentrations at either end of a restricted-diffusion cell [13]; Newman et al. have used UV/visible spectrophotometry [22] and interferometry [4] to measure concentrations directly.

It has been observed that potentiometric techniques tend to yield higher diffusivities $[22,23]$. This is natural in light of the inaccuracy that the approximation in equation 9 can induce in data processing. As concentration polarization decreases during the relaxation period, the difference between the real experimental $\Delta y$ (denoted as $\left.\Delta y_{\text {real }}\right)$ and $\Delta y$ obtained from $\Delta U$ through equation 9 (denoted as $\Delta y_{\text {appr }}$, which is generally higher than $\Delta y_{\text {real }}$ ) gets smaller. The magnitude of the slope of $\ln \left(\Delta y_{\text {appr }}\right) v s$. time is higher than that of $\ln \left(\Delta y_{\text {real }}\right) v s$. time, leading to a higher apparent diffusivity. Nevertheless, the inaccuracy brought by applying equation 9 is usually minimal, since the approximation that composition differences are small is often reasonable after long relaxation times.

\subsection{Galvanostatic polarization}

Galvanostatic-polarization measurements track composition relaxations associated with diffusion close to the electrodes in a polarization cell. The underlying theory relies on data collected before the diffusion boundary layers induced by a current pulse come into contact, when migration and diffusion control the developing concentration profile. In this regime, interelectrode distance is unimportant; regions near the electrodes can be treated as semiinfinite.
Data processing requires precise knowledge of the OCP immediately after a current pulse. In practice, some capacitive relaxation - neglected by the theory - occurs at very short times after the pulse. Thus, researchers have almost exclusively used an extrapolation method [11, 21], in which the OCP during relaxation is recorded as a function of a transformed time variable $\tilde{\tau}$, defined as

$$
\tilde{\tau}=\frac{\sqrt{\tau_{\text {pulse }}}}{\sqrt{\tau}+\sqrt{\tau-\tau_{\text {pulse }}}} .
$$

This time variable is conveniently restricted between 0 and 1 , where 0 corresponds to time infinity and 1 to the initial instant of relaxation after $\tau_{\text {pulse }}=\mathscr{D} \chi t_{\text {pulse }} / L^{2}$. With the linear approximation from equation 9 , the solution of the transient diffusion equation with $\beta=0$ predicts that $\Delta U$ should depend linearly on $\tilde{\tau}$. Thus $\Delta U$ vs. $\tilde{\tau}$ data can be extrapolated to $\left.\Delta U\right|_{\tilde{\tau}=1}$, yielding the diffusion potential immediately after the pulse. A plot of $\left.\Delta U\right|_{\tilde{\tau}=1}$ vs. $i \sqrt{t}$ should also be linear; if $\alpha=0$ as well, its slope $M$ is [11]

$$
M=\left.\frac{4\left(1-t_{+}^{0}\right)}{z_{+} \nu_{+} F\langle c\rangle \sqrt{\pi D}}\left(\frac{d U}{d \ln c}\right)\right|_{\langle c\rangle} .
$$

Note that in terms of the particle-fraction basis,

$$
\begin{aligned}
\left.\frac{1}{\langle c\rangle}\left(\frac{d U}{d \ln c}\right)\right|_{\langle c\rangle} & \\
& =\left.\frac{\left[\bar{V}_{0}-\left(\nu \bar{V}_{0}-\bar{V}_{\mathrm{e}}\right)\langle y\rangle\right]^{2}}{\bar{V}_{0}\langle y\rangle}\left(\frac{d U}{d \ln y}\right)\right|_{\langle y\rangle}
\end{aligned}
$$

Insertion of equations 7 and 21 into equation 20 gives a relationship among the transference number, diffusivity, thermodynamic factor, and partial molar volumes.

Application of the linearization in equation 9 has significant impact on measurements by galvanostatic polarization, which involve setting up steep concentration gradients in boundary layers. Many groups have observed curvature in $\Delta U$ vs. $\tilde{\tau}$ data as $\tilde{\tau}$ approaches $1[12,21]$. Experimentally observed curvature often occurs over much longer time scales than the magnitudes of double-layer capacitances can justify [12]. The time constant for doublelayer relaxation, $t_{\mathrm{dl}}$, scales as $t_{\mathrm{dl}} \sim \epsilon L /(2 \kappa \lambda)$, where $\epsilon$ is the dielectric permittivity of the solution and $\lambda$, its Debye length. For nonaqueous electrolytes at the concentrations discussed here, $t_{\mathrm{dl}} / L$ is typically of the order of $1 \mathrm{sm}^{-1}$ at room temperature. Since even the widest electrode spacings used in practice are a few centimeters, double-layer relaxation is expected to occur within a fraction of a second after the termination of a current pulse.

Replacing equation 9 with equation 8 can rationalize the prior observations of non-capacitive curvature in $\Delta U$ vs. $\tilde{\tau}$ data. Figure 2 shows a series of simulated relaxation curves after a $500 \mathrm{~s}$ galvanostatic polarization of $0.85 \mathrm{M}$ lithium hexafluorophosphate $\left(\mathrm{LiPF}_{6}\right)$ in propylene carbonate $(\mathrm{PC})$, in a planar, symmetric plating/stripping cell of 1 $\mathrm{cm}$ length with lithium-metal electrodes. (Both electrode 


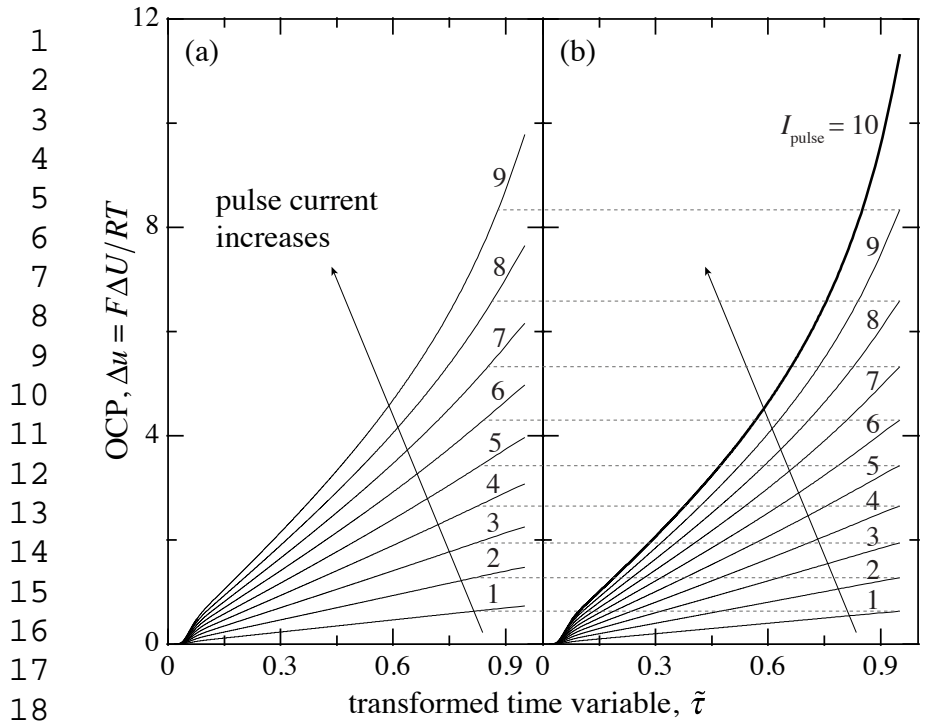

19

20

21

22

23

24

25

26

27

28

29

30

31

32
20 along with correct values of $D$ and $d U / d \ln c$, produces low transference numbers. This could explain the negative values of $t_{+}^{0}$ observed by Doeff et al. [17].

\subsection{Solute-volume effects}

Two solute-volume effects can be significant in moderately concentrated non-aqueous electrolytes [16]: the 'excluded-volume effect' arises from volume redistribution that accompanies concentration changes, and is quantified by $\alpha$; 'Faradaic convection' occurs when interfacial electrochemical reactions induce bulk flow, and is measured by $\beta$. Substantial measurement errors can arise when pulse/relaxation data are analyzed with a theory that ignores solutevolume effects - roughly speaking, $\alpha$ and $\beta$ respectively measure the fractional errors in the diffusion potential and the limiting current.

Currents higher than the limiting current are often desirable for galvanostatic polarization because they induce large concentration differences over short distances. In a cell polarized at the limiting current, the salt concentration is driven to zero at one of the electrodes only when the steady state is achieved (after a very long time). When a higher current is applied, complete consumption of the active species at an electrode takes a finite amount of time, known as 'Sand's time' (or, the 'transition time') [27-29]. In a solution where solute-volume effects are negligible, Sand's time $t_{\text {sand }}$ (or, dimensionlessly, $\tau_{\text {sand }}$ ) relates to the current through

$$
\tau_{\text {sand }}^{\infty}=\frac{D t_{\text {sand }}^{\infty}}{L^{2}}=\frac{\pi}{16 I^{2}},
$$

where the superscript $\infty$ indicates a quantity at infinite dilution (in the limit $\alpha, \beta \rightarrow 0$ ). Equation 22 agrees with the formula reported by Bard and Faulkner [18] (equation 8.2.14, p. 310); it is somewhat extended, however, because Bard and Faulkner neglect migration, assuming a wellsupported electrolyte for which $t_{+}^{0} \approx 0$. The derivation of equation 22 assumes a semi-infinite diffusion domain; its validity is restricted to times before the diffusion boundary layers meet.

Although there are a few notable exceptions, Faradaic convection usually increases the current tolerance of electrolytic solutions [16]. The effect can be seen by comparing Figure 2(b), which accounts for $\beta$, with Figure 2(a), where $\beta=0$. The highest pulse current adopted in generating Figure $2(\mathrm{~b})$ is $I_{\text {pulse }}=10$; in the limit $\alpha, \beta \rightarrow 0$, a current of this magnitude cannot be sustained, because the specified pulse duration is greater than Sand's time. Generally, solutions with Faradaic convection will have longer Sand's times when $\beta>0$.

In diffusivity and transference-number measurements, establishing a composition distribution that can be tracked and predicted by equations 10 and 16 through 18 is essential. The polarization process should be strictly controlled to prevent the complete consumption of a limiting reactant at either electrode - a process that can be guided by 


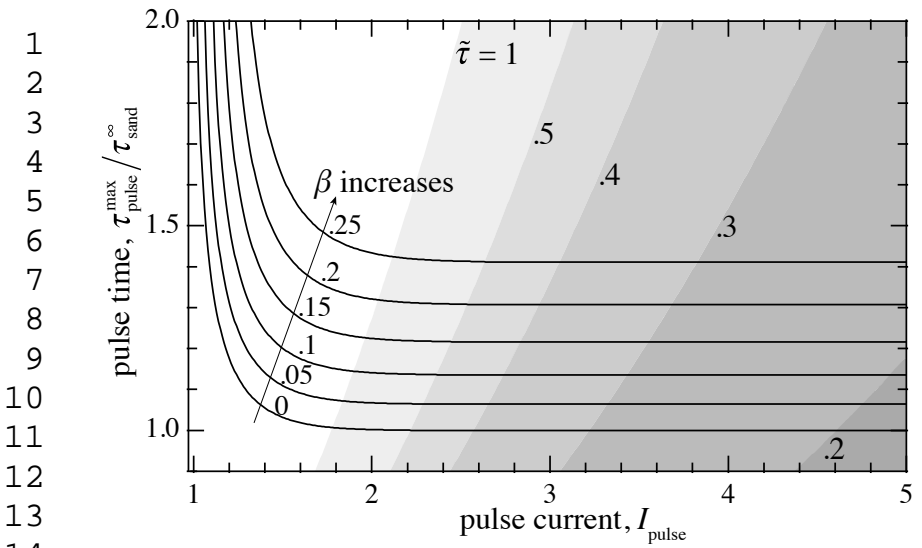

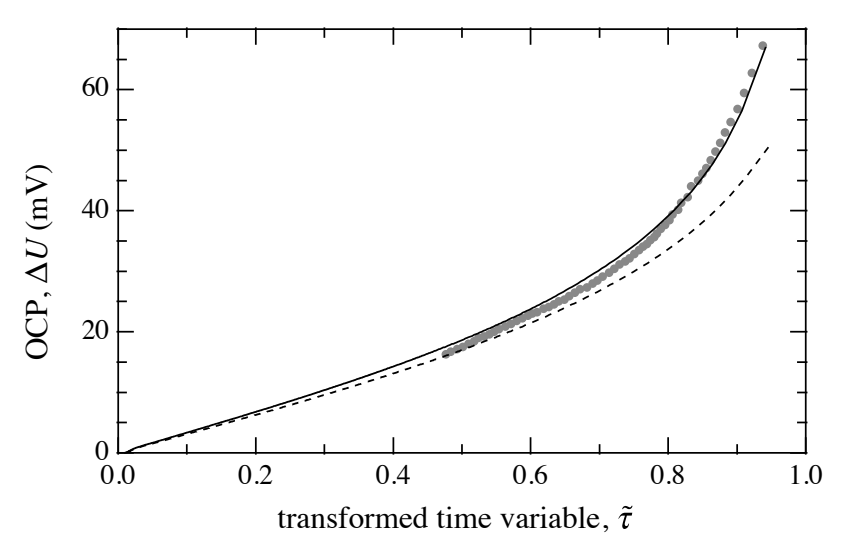

Figure 4: OCP vs. $\tilde{\tau}$ for $2.24 \mathrm{M} \mathrm{LiPF}_{6}$ in a mixed PC/EC/DMC solvent. Experimental data (gray dots) are reproduced from Valøen and Reimers [12]. The black solid curve is simulation output generated with $D=1.55 \times 10^{-6} \mathrm{~cm}^{2} \mathrm{~s}^{-1}$; the black dashed curve shows data for $D=1.8 \times 10^{-6} \mathrm{~cm}^{2} \mathrm{~s}^{-1}$, as reported by Valøen and Reimers.

contours and 1 on an OCP vs. $\tilde{\tau}$ curve should be used for data analysis with the semi-infinite transport equations.

In addition to changing the apparent current tolerance of a solution, solute-volume effects also influence the diffusion potential. The OCP during transient relaxation is lower when solute-volume effects are involved than when they are not, as shown by the horizontal tie lines connecting curves at the same current in Figures 2(a) and 2(b). In most electrolytic systems of practical interest, solutevolume effects suppress the diffusion potential during diffusivity and transference-number measurements.

\subsection{Data fitting}

The governing system from section 3.2 was solved to simulate the OCP vs. $\tilde{\tau}$ data Valøen and Reimers gathered for a $2.24 \mathrm{M} \mathrm{LiPF}_{6}$ solution in a mixed $\mathrm{PC} /$ ethylene carbonate/dimethyl carbonate solvent [12], which are reproduced in Figure 4. The data show curvature at the beginning of the relaxation ( $\tilde{\tau}$ close to 1 ), when the concentration gradient across the cell is largest and the assumed proportionality between $\Delta U$ and $\Delta y$ in equation 9 is least accurate. Valøen and Reimers performed a concentrationcell measurement to obtain $d U / d \ln c=0.017 \mathrm{~V}$, implemented a Hittorf experiment to establish that $t_{+}^{0}=0.38$, and then used galvanostatic polarization to get the diffusivity with equation 20. By making a linear fit of the data between $\tilde{\tau}=0.5$ and $\tilde{\tau}=0.8$ and extrapolating to $\tilde{\tau}=1$, they concluded that $D=1.8 \times 10^{-6} \mathrm{~cm}^{2} \mathrm{~s}^{-1}$.

Our simulations, based on equations 10 and 16 through 18, fit all of Valøen and Reimers's experimental data well with a Fickian diffusivity of $D=1.55 \times 10^{-6} \mathrm{~cm}^{2} \mathrm{~s}^{-1}$. The complete set of properties used for fitting the experimental data is listed in Table 2. (Details of the partial-molar volume measurements are provided in the Supplementary Information that accompanies this article.) When adopting the higher diffusivity reported by Valøen and Reimers, the model that accounts for solute-volume effects systematically underpredicts the experimental voltages. In general, 


\begin{tabular}{r|ccc|cc|}
\cline { 2 - 6 } 1 & prop. & value & ref. & prop. & value \\
\cline { 2 - 6 } 2 & $\kappa$ & $1.0 \mathrm{~S} \mathrm{~m}^{-1}$ & {$[12]$} & $\mathscr{D}_{+-}$ & $2.1 \times 10^{-11} \mathrm{~m}^{2} \mathrm{~s}^{-1}$ \\
3 & $D$ & $1.55 \times 10^{-10} \mathrm{~m}^{2} \mathrm{~s}^{-1}$ & $\diamond$ & $\mathscr{D}_{0+}$ & $1.8 \times 10^{-10} \mathrm{~m}^{2} \mathrm{~s}^{-1}$ \\
4 & 0.38 & {$[12]$} & $\mathscr{D}_{0-}$ & $3.0 \times 10^{-10} \mathrm{~m}^{2} \mathrm{~s}^{-1}$ \\
5 & $t_{+}^{0}$ & & $\bar{V}_{0}$ & $78.1 \mathrm{~cm}^{3} \mathrm{~mol}^{-1}$ \\
6 & $\bar{V}_{0}$ & $78.1 \mathrm{~cm}^{3} \mathrm{~mol}^{-1}$ & $\diamond$ & $\bar{V}_{0}$ & \\
7 & $\bar{V}_{\mathrm{e}}$ & $55.5 \mathrm{~cm}^{3} \mathrm{~mol}^{-1}$ & $\diamond$ & $\bar{V}_{+}$ & $34.4 \mathrm{~cm}^{3} \mathrm{~mol}^{-1}$ \\
8 & & & & $\bar{V}_{-}$ & $21.1 \mathrm{~cm}^{3} \mathrm{~mol}^{-1}$ \\
9 & & & & & \\
10 & $\frac{d U}{d \ln c}$ & $0.017 \mathrm{~V}$ & {$[12]$} & $\chi$ & 0.68 \\
\hline 1 & & & & &
\end{tabular}

Table 2: Properties of $2.24 \mathrm{M} \mathrm{LiPF}_{6}$ in the mixed PC/EC/DMC were used for simulations, and were calculated from those on the left. Diamonds indicate properties reported for the first time here.

fitting relaxation curves with models that neglect solutevolume effects can result in low apparent values of $M$, leading to artificially high $D$ or $t_{+}^{0}$ values.

\section{Conclusion}

Potentiometric restricted-diffusion and galvanostaticpolarization experiments have been analyzed to address measurement issues that may lead to inaccurate property determination for concentrated binary electrolytic solutions. The methods for measuring electrolyte thermodynamic factor, diffusivity and transference number in simple binary electrolytes are developed on the basis of a single equation that relates open-circuit potential to solution composition. Using a particle-fraction basis for compositiondependent properties brings mathematical convenience, simplifies the physical relationships needed in the ideal-solution limit, and helps avoid systematic errors that may be induced by temperature or pressure fluctuations.

The assumption that the polarization-induced opencircuit potential drop $\Delta U$ is proportional to the extent of composition polarization $\Delta y$ is only accurate when $\Delta y$ is quite small. When $\Delta y$ gets too large, a measured value of $\Delta U$ will correspond to an actual $\Delta y$ lower than the one predicted by the linear approximation. The curvature observed by many research groups in OCP vs. $\tilde{\tau}$ curves from galvanostatic-polarization experiments is caused primarily by the nonlinear relation between $\Delta U$ and $\Delta y$. Data fits using curved OCP vs. $\tilde{\tau}$ data may result in low - or even negative - transference numbers.

Solute-volume effects lead to other issues, changing the current tolerances of electrolytic solutions and also impacting their diffusion potentials. Sand's time was recalculated for electrolytic solutions with different Faradaicconvection numbers, showing the impacts of finite interelectrode distance as well as reaction-induced convection. Galvanostatic-polarization experiments should be designed and implemented so that the current pulse lasts no longer than Sand's time, and so that enough data can be collected before the boundary layers caused by the pulse meet. Ne- glecting solute-volume effects when analyzing data can lead to apparently elevated diffusion coefficients or transference numbers.

\section{Acknowledgments}

This work was supported by the Joint Center for Energy Storage Research, an Energy Innovation Hub funded by the U.S. Department of Energy, Office of Science, Basic Energy Sciences. solution studied by Valøen and Reimers [12]. Properties on the right

\section{References}

[1] L. Onsager, Theories and problems of liquid diffusion, Annals of the New York Academy of Sciences 46 (1945) 241-265.

[2] J. Newman, K. E. Thomas-Alyea, Electrochemical Systems, 3rd ed., Wiley Interscience, 2004.

[3] A. Nyman, M. Behm, G. Lindbergh, Electrochemical characterisation and modelling of the mass transport phenomena in $\mathrm{LiPF}_{6}$-EC-EMC electrolyte, Electrochimica Acta 53 (2008) $6356-6365$.

[4] J. Newman, T. W. Chapman, Restricted diffusion in binary solutions, AIChE Journal 19 (1973) 343-348.

[5] M. S. Ding, Electrolytic conductivity and glass transition temperature as functions of salt content, solvent composition, or temperature for $\mathrm{LiPF}_{6}$ in propylene carbonate + diethyl carbonate, Journal of Chemical \& Engineering Data 48 (2003) 519-528.

[6] L. Edman, M. M. Doeff, A. Ferry, J. Kerr, L. C. De Jonghe, Transport properties of the solid polymer electrolyte system $\mathrm{P}(\mathrm{EO})_{n}$ LiTFSI, The Journal of Physical Chemistry B 104 (2000) 3476-3480.

[7] I. Hodge, M. Ingram, A. West, Impedance and modulus spectroscopy of polycrystalline solid electrolytes, Journal of Electroanalytical Chemistry and Interfacial Electrochemistry 74 (1976) $125-143$.

[8] S. Mikhailenko, M. Guiver, S. Kaliaguine, Measurements of PEM conductivity by impedance spectroscopy, Solid State Ionics 179 (2008) 619-624.

[9] P. M. Sipos, G. Hefter, P. M. May, Viscosities and densities of highly concentrated aqueous $\mathrm{MOH}$ solutions $\left(\mathrm{M}^{+}=\mathrm{Na}^{+}\right.$, $\left.\mathrm{K}^{+}, \mathrm{Li}^{+}, \mathrm{Cs}^{+},\left(\mathrm{CH}_{3}\right)_{4} \mathrm{~N}^{+}\right)$at $25.0{ }^{\circ} \mathrm{C}$, Journal of Chemical \& Engineering Data 45 (2000) 613-617.

[10] A. A. Shinkle, A. E. S. Sleightholme, L. T. Thompson, C. W. acetylacetonate flow batteries, Journal of Power Sources 238 (2014) 1299-1305.

[11] Y. Ma, M. Doyle, T. F. Fuller, M. M. Doeff, L. C. DeJonghe, J. Newman, The measurement of a complete set of transport properties for a concentrated solid polymer electrolyte solution, Journal of The Electrochemical Society 142 (1995) 1859-1868.

[12] L. O. Valøen, J. N. Reimers, Transport properties of $\mathrm{LiPF}_{6}{ }^{-}$ based Li-ion battery electrolytes, Journal of The Electrochemical Society 152 (2005) A882-A891.

[13] H. S. Harned, D. M. French, A conductance method for the determination of the diffusion coefficients of electrolytes, Annals of the New York Academy of Sciences 46 (1945) 267-281.

[14] A. Ferry, M. M. Doeff, L. C. DeJonghe, Transport property measurements of polymer electrolytes, Electrochimica Acta 43 (1998) 1387-1393.

[15] J. Zhao, L. Wang, X. He, C. Wan, C. Jiang, Determination of lithium-ion transference numbers in $\mathrm{LiPF}_{6}-\mathrm{PC}$ solutions based on electrochemical polarization and NMR measurements, Journal of The Electrochemical Society 155 (2008) A292-A296.

[16] J. Liu, C. W. Monroe, Solute-volume effects in electrolyte transport, Electrochimica Acta 135 (2014) 447-460. Monroe, Solvents and supporting electrolytes for vanadium 
1 [17] M. M. Doeff, L. Edman, S. E. Sloop, J. Kerr, L. C. DeJonghe, Transport properties of binary salt polymer electrolytes, Journal of Power Sources 89 (2000) 227-231.

[18] A. J. Bard, L. R. Faulkner, Electrochemical Methods: Fundamentals and Applications, 2nd ed., Wiley, 2001.

[19] M. Ciosek, M. Marcinek, G. Żukowska, W. Wieczorek, Lithium transference number measurements and complex abilities in anion trapping triphenyloborane-poly(ethylene oxide) dimethyl ether-lithium trifluoromethanesulfonate composite electrolyte, Electrochimica Acta 54 (2009) 4487-4493.

[20] S. Zugmann, M. Fleischmann, M. Amereller, R. Gschwind, H. Wiemhöfer, G. H. J., Measurement of transference numbers for lithium ion electrolytes via four different methods, a comparative study, Electrochimica Acta 56 (2011) 3926-3933.

[21] H. Hafezi, J. Newman, Verification and analysis of transference number measurements by the galvanostatic polarization method, Journal of The Electrochemical Society 147 (2000) 3036-3042.

[22] S. G. Stewart, J. Newman, The use of UV/vis absorption to measure diffusion coefficients in $\mathrm{LiPF}_{6}$ electrolytic solutions, Journal of The Electrochemical Society 155 (2008) F13-F16.

[23] A. Mehrotra, V. Srinivasan, Transport property measurements for $\mathrm{LiPF}_{6}$ in EC:DEC (1:1), The 224th Electrochemical Society Meeting Abstract \# 1152 (2013).

[24] T. Nishida, K. Nishikawa, Y. Fukunaka, Diffusivity measurement of $\mathrm{LiPF}_{6}, \mathrm{LiTFSI}, \mathrm{LiBF}_{4}$ in PC, ECS Transactions 6 (2008) 1-14.

[25] S. G. Stewart, Determination of Transport Properties and Optimization of Lithium-Ion Batteries, Ph.D. thesis, University of Canifornia, 2007.

[26] S. Stewart, J. Newman, Measuring the salt activity coefficient in lithium-battery electrolytes, Journal of The Electrochemical Society 155 (2008) A458-A463.

[27] R. Cvetković, B. N. Popov, H. A. Laitinen, Electrochemical reduction of molybdate in the presence of zinc chloride in molten lithium chloridepotassium chloride eutectic, Journal of The Electrochemical Society 122 (1975) 1616-1619.

[28] B. N. Popov, H. A. Laitinen, Electrochemical reduction of chromate in the presence of nickel chloride in molten lithium chloridepotassium chloride eutectic, Journal of The Electrochemical Society 117 (1970) 482-484.

[29] B. N. Popov, H. A. Laitinen, Electrochemical reduction of molybdenum(VI) compounds in molten lithium chloridepotassium chloride eutectic, Journal of The Electrochemical Society 120 (1973) 1346-1350. 
Click here to download Supplementary Materials: supplementary materials_revise_final.pdf

Supplementary Materials for Publication
Click here to download Supplementary M

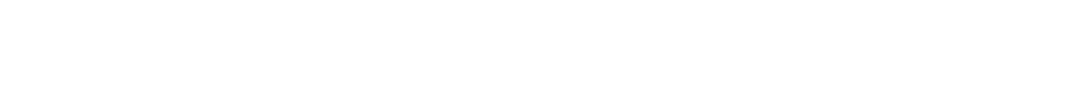

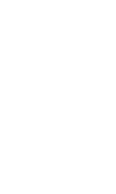

(1) 
Click here to download Supplementary Materials: property measurements_revise_final marked.pdf

Marked manuscript
Click here to downlc

upplementary Materials: property measurements_revise_final marked.pdf 
LaTeX Source Files
Click here to download LaTeX Source Files: Latex files.zip

LaTeX Source Files
Click here to download LaTeX Source Files: Latex files.zip

Click here to download LaTeX Source Files: Latex files.zip

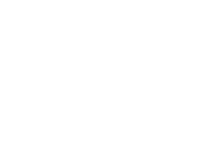

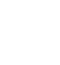

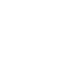

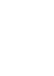
(1) (1) (1) (1) (1) (1) (1) (1) . . . . . . . . . . . .

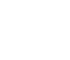

